\title{
Asthma and chronic sinusitis: one disease for two organs
} Asthme et sinusite chronique: une maladie pour deux organes

\author{
S. Duong-Quy \\ Collège de Médecine de Lam Dong. Vietnam \\ Département de Pneumologie et Immuno-Allergologie. Université Penn State. USA \\ Service des Explorations Fonctionnelles Respiratoires. Hôpital Cochin. Université Paris Descartes. France
}

La sinusite chronique est souvent associée à l'asthme. Elle est présente dans la quasi-totalité des patients présentant des symptômes de l'asthme. L'association fréquente de l'asthme et la sinusite a été noté depuis plusieurs années. Il y a environ 50\% des personnes atteintes $\mathrm{d}^{\prime}$ asthme modéré ou sévère ont aussi une sinusite chronique. En plus, jusqu'à 80 à $90 \%$ des enfants et des adolescents souffrant d'asthme ont également le symptôme nasal, et la moitié des patients souffrant d'asthme ont preuve d'imagerie de la sinusite [1]. Le résultat des études précédentes on montrées que la prévalence de la sinusite chronique a été aussi élevée que 40 à $60 \%$ chez les enfants atteints de l'asthme [2,3]. En Taiwan, $10,4 \%$ des enfants ont l'asthme [4] et la moitié d'entre eux ont également concomitante sinusite [5].

La forte prévalence de l'association entre l'asthme et la sinusite peut être due à l'état inflammation de l'ensemble des muqueuses respiratoires chez les terrains allergiques qui sont souvent retrouvés dans cette population. Comme dans l'asthme, la sinusite peut être déclencher par des allergènes ou des infections. Les déclencheurs courants de la sinusite sont: une infection virale ou bactérienne, la pollution atmosphérique et la fumée de la cigarette, les pneumallergènes, l'air froid ou sec.

De nombreuses études ont montré un lien entre les infections des sinus et l'asthme. Les patients asthmatiques ayant la concomitante sinusite ont tendance à avoir des symptômes plus sévères d'asthme, peuvent avoir des crises $d^{\prime}$ asthme plus sévère et sont plus susceptibles d'avoir des troubles du sommeil.
Cependant, les risques de développer une sinusite peuvent ne pas être la même pour tous les patients asthmatiques. L'association de la sinusite à l'asthme est plus fréquente chez les femmes que les hommes et plus fréquente chez les européens que les autres groupes raciaux. De plus, le reflux gastroœsophagien (RGO) et le tabagisme peuvent accroître le risque de développer la sinusite chez le sujet asthmatique. Chez les personnes atteintes d'asthme sévère, la sinusite semble aggraver les symptômes $\mathrm{d}$ 'asthme et les rend difficile à contrôler. Pourtant, certains cliniciens considèrent que la sinusite est un problème banal et n'a pas besoin d'être traité de façon spécifique en dehors du traitement spécifique de la rhinite allergique associée.

Dans le numéro 20 du JFVP 2016, Gagara Issoufou et collaborateurs [6] ont montré que la fréquence de l'association de l'asthme et de la sinusite chronique retrouvée sur le nombre total des consultants chez 117 patients asthmatiques durant la période de 6 mois (Décembre 2013 - Mai 2014) était de 10,2\%. Il s'agissait d'une étude prospective, transversale et descriptive à la Clinique Médicale Lacouroussou (CML) et à l'Hôpital National de Lamordé (HNL) et au niveau de la consultation pneumo-allergologique. Les auteurs ont montré qu'au moment de la consultation, $87 \%$ des patients n'avaient pas un traitement de fond, expliqué par une méconnaissance du traitement de fond par des professionnels de la santé, et $22,1 \%$ des patients avaient un asthme contrôlé. Dans cette étude, la toux et la dyspnée ont été les symptômes retrouvés tant dans l'asthme que dans la sinusite en phase aigüe alors que l'obstruction est spécifique de la sinusite.

Auteur correspondant: Pr. Sy DUONG-QUY. Collège de Médecine de Lam Dong - Vietnam. Département de Pneumologie et d'Immuno-Allergologie. Université Penn State - USA. Service des Explorations Fonctionnelles Respiratoires. Hôpital Cochin. Université Paris Descartes - France. E-mail: sduongquy.jfop@gmail.com 
Les auteurs suggèrent que cette association fréquente entre symptômes de sinusite et l'asthme peut s'expliquer par plusieurs facteurs: la présence d'un réflexe rhino-bronchique, le passage direct des médiateurs de l'inflammation des voies aériennes supérieures aux voies aériennes inférieures, suggérant l'existence d'un mécanisme physiopathologique commun au niveau des voies aériennes et la fréquence des infections bactériennes des sinus para nasaux chez l'asthmatique qui peuvent affecter les poumons et entraîner des infections respiratoires.

\section{CONFLIT D'INTÉRÊT}

Aucun.

\section{RÉFÉRENCES}

1. Corren J, Rachelefsky GS. Interrelationship between sinusitis and asthma. Immunol Allergy Clin North Am 1994; 14:171-183.

2. Berman S, Mathison DA, Stevenson DD, et al. Maxillary sinusitis and bronchial asthma: correlation of roentgenograms, cultures and thermograms. J Allergy Clin Immunol 1974; 53:311-318.

3. Newman LJ, Platts-Mills TAE, Phillips D, et al. Chronic sinusitis: relationship of computed tomographic findings to allergy, asthma, and eosinophilia. JAMA 1994; 271:363-367.
Les auteurs ont conclu que l'association de l'asthme et la sinusite rend le contrôle de l'asthme difficile, ainsi tout patient asthmatique doit bénéficier $\mathrm{d}^{\prime}$ un bilan ORL complet afin de diagnostiquer une sinusite ou autre comorbidité associée. Un traitement de la sinusite associé à un traitement fond de l'asthme avec un suivi régulier permet un meilleur contrôle de l'asthme et avec tous les problèmes causés par l'asthme, avoir la sinusite peut être difficile à gérer. La sinusite a été associée à des cas plus graves d'asthme.
4. Huang JL, Chen LC, Kuo ML, et al. Exposure to a high concentration of mite allergen in early infancy is a risk factor for developing atopic dermatitis. Pediatr Allergy Immunol 2001; 12:11-16.

5. Huang JL, Lin TY, Wang KF. Sinusitis and bronchial asthma in children. Acta Paediatr Sin 1995; 36:20-23.

6. Gagara Issoufou MA, Assao Neino MM, Inne Pierre M, Adambounou TS, Soumana AZ, Bako M, Maizoumbou D. The association of asthma and sinusitis in Niamey. $J$ Fran Viet Pneu 2016;20(7):42-46. 